\title{
A Case of Internal Carotid Artery Thrombosis associated with Thalidomide Administration in Multiple Myeloma
}

\author{
Maria Angela Grima, David James Camilleri \\ Department of Health, Mater Dei Hospital, Triq Dun Karm, L-Imsida, Malta
}

Received: $18 / 02 / 2016$

Accepted: 09/03/2016

Published: 04/05/2016

\begin{abstract}
How to cite this article: Grima MA, DJ Camilleri. A case of internal carotid artery thrombosis associated with thalidomide administration in multiple
\end{abstract} myeloma. EJCRIM 2016;3:doi:10.12890/2016_000410

Conflicts of Interests: The Authors declare that there are no competing interests.

This article is licensed under a Commons Attribution Non-Commercial 4.0 License

\section{ABSTRACT}

Thalidomide is an effective chemotherapeutic agent used to achieve remission in multiple myeloma. However, its administration is associated with several adverse effects including venous thromboembolism, while arterial thrombosis has also, although rarely, been described in the literature. We report a case of internal carotid artery occlusion within 1 week of starting thalidomide with prophylactic low molecular weight heparin in a patient who had no other prothrombotic risk factors. It is not known why this complication occurs despite the administration of anticoagulant prophylaxis. The role of factor VIII, von Willebrand factor antigen levels and fibrinogen in multiple myeloma patients should be studied in order to determine if these factors should be targeted in future prophylactic treatment.

\section{LEARNING POINTS}

- Thalidomide increases risk of arterial thrombosis in multiple myeloma (MM), even if no other procoagulant risk factors are present.

- All patients starting thalidomide must be assessed before commencing venous thromboprophylaxis, although its efficacy in preventing arterial thrombosis has not yet been proven.

- Further research is required to target anti-thrombotic factors in order to prevent arterial thrombosis in patients with MM being treated with thalidomide and its derivatives.

\section{KEYWORDS}

Multiple myeloma; thalidomide; arterial thrombosis; cerebrovascular accident.

\section{CASE REPORT}

A 45-year-old woman presented with mild lethargy, fatigue and generalised myalgia, but had an otherwise unremarkable history. Upon investigation, she was found to be mildly cytopenic with a haemoglobin level of $11.3 \mathrm{~g} / \mathrm{dl}$ and a platelet count of $69 \times 10^{9} / \mathrm{l}$. Her peripheral blood film revealed a left shift in the neutrophils and erythrocyte anisopoikilocytosis with occasional teardrop red cells. Haematinics, an autoimmune antibody screen and viral screens were negative. Further investigations ultimately confirmed the presence of multiple myeloma (MM).

\section{METHODS AND PROCEDURES}

Serum protein electrophoresis demonstrated hypogammaglobulinaemia and an initial low level of paraprotein which later increased to $3 \mathrm{~g} / \mathrm{l}$. 
This was confirmed to be an IgG lambda monoclonal band on immunofixation. The patient had markedly elevated serum free light chains, with a kappa to lambda ratio of 0.001 (normal range 0.26-1.65).

MM was confirmed by bone marrow trephine biopsy. It revealed hypercellularity with a nodular infiltrate of plasma cells, the majority of which expressed syndecan-1 (CD138), together with lymphoplasmacytoid cells concurrently expressing CD138 and CD20. The aspirate was aparticulate, with occasional precursors of erythropoiesis and myelopoiesis, while megakaryocytes were virtually absent, which was most likely due to the poor quality of the aspirate.

Spinal magnetic resonance imaging (MRI) showed diffuse vertebral body marrow infiltration which enhanced upon contrast administration. However, no lesions were detected on skeletal survey.

The patient was started on thalidomide $100 \mathrm{mg}$ on alternate days, cyclophosphamide $500 \mathrm{mg}$ once weekly and dexamethasone $40 \mathrm{mg}$ daily on days 1-4 and 12-15 according to the cyclophosphamide, thalidomide, dexamethasone (CTD) protocol, with daily low molecular weight heparin (LMWH) $40 \mathrm{mg}$ as prophylaxis for venous thromboembolism (VTE).

Within 1 week the patient presented with sudden onset right hemiparesis and facial paralysis, together with expressive aphasia. An isoechoic acute thrombus in the left carotid bulb extending into the proximal segment of the left internal carotid artery was confirmed by Doppler ultrasonography, while an MRI showed infarction of the left cerebral hemisphere.

Our subject's medical history was unremarkable. There was no evidence of atrial fibrillation, hypertension, hyperlipidaemia or diabetes mellitus, and the vasculitic screen was negative. She was investigated for hereditary causes of thrombophilia but factor $V$ Leiden, homocystinuria MTFR gene mutations and prothrombin factor mutations were all absent. Moreover, the international normalised ratio, prothrombin time, antithrombin III activity, protein C activity and antigen, protein S activity and antigen, and free protein S function were all within normal range. Lupus anticoagulant was also negative. The only abnormal result was an activated partial thromboplastin time ratio of 0.84 (normal range $0.9-1.1$ ).

Thalidomide and cyclophosphamide were withdrawn and later replaced with bortezomib and dexamethasone. Low-dose anti-platelet therapy and a rehabilitation programme led to significant improvement, although the patient still has residual right-sided hemiparesis and expressive aphasia.

\section{DISCUSSION}

Various chemotherapeutic regimens are used as first line therapy in MM, with the CTD protocol commonly used to achieve remission ${ }^{[1]}$. Although thalidomide has improved survival, it is associated with high morbidity. Recent data have shown that around $35 \%$ of all reported thalidomide-associated thromboembolic episodes were arterial in nature, with $54.2 \%$ of these involving the coronary arteries and $19.8 \%$ involving the cerebral vasculature ${ }^{[2]}$.

Arterial thrombosis associated with thalidomide administration is a rare complication, but it is even more unusual for such an event to occur on a background of anticoagulation, as in our patient. The pathogenesis of arterial thrombosis induced by this agent is poorly recognized. The risk for this complication is highest in the first year of treatment and also when thalidomide is administered with other cytotoxic chemotherapeutic agents ${ }^{[3]}$, as was the case in our subject. The main risk factors for thrombosis were malignancy and the combination of thalidomide with high-dose dexamethasone and an alkylating agent, automatically classifying our subject as high risk.

In such high risk settings, the guidelines set by the Haemato-oncology Task Force of the British Committee for Standards in Haematology (BCSH) and the UK Myeloma Forum suggest the use of prophylactic LMWH or full-dose warfarin (to reach a target INR of 2-3), since the efficacy of aspirin (81-325 mg/day) is largely uncertain and it is only considered if no risk factors for VTE are present (grade B2 evidence) ${ }^{[4]}$. The use of low-dose ( $20 \mathrm{mg} / \mathrm{day}$ ) LMWH or vitamin $\mathrm{K}$ antagonists is recommended if patients have renal disease, while full-dose (40 mg/ day) LMWH would be more useful in thrombocytopenic patients since it has a lower risk of haemorrhage. Nonetheless, the choice between these two prophylactic modalities is left to the physician's discretion, since patient compliance with regular INR monitoring and the risks of bleeding must both be considered. Despite reducing the rate of VTE from $20 \%$ to $10 \%$ in high risk cases, their efficacy regarding arterial thrombosis prophylaxis is still uncertain ${ }^{[5]}$.

Most reports in the literature included patients not on anticoagulation who later experienced various arterial and venous thrombotic events $^{[6-12]}$. However, to the authors' knowledge, only Scarpace ${ }^{[13]}$ et al. have reported two such events which occurred in patients receiving anticoagulation with aspirin and warfarin, respectively. The HOVON 50 study described two patients who experienced an arterial thrombotic event despite receiving LMWH thrombosis prophylaxis during thalidomide, doxorubicin and dexamethasone therapy ${ }^{[14]}$. Palumbo et al. conducted a randomised study which compared the use of prophylactic acetylsalicylic acid (ASA; $100 \mathrm{mg} / \mathrm{day}$ ), fixed low-dose warfarin (1.25 $\mathrm{mg} /$ day) and LMWH (enoxaparin $40 \mathrm{mg} /$ day) in patients with MM treated with thalidomide. About $7 \%$ of patients had serious thrombotic events, ranging from acute cardiovascular events to sudden death during the first 6 months of treatment. Overall, $8 \%$ were on warfarin, $6 \%$ 
on ASA and 5\% on LMWH. These three antithrombotic drugs showed comparable incidence of thromboembolic complications in patients with MM. However, warfarin was shown to be less effective than LMWH in the elderly population ${ }^{[15] .}$

Therefore, the choice between aspirin, vitamin $\mathrm{K}$ antagonists and heparin very much depends on the clinical scenario. The patient-related risk factors for thromboembolic events, the type of chemotherapeutic protocol (especially when thalidomide is combined with doxorubicin, dexamethasone or another chemotherapeutic agent), the risks of haemorrhage and cost effectiveness must be all considered. Once all these are evaluated, the appropriate anticoagulant strategy can be chosen by following the guidelines in order to optimise patient outcome. In this case, the short interval between the initiation of thalidomide and the acute event, together with the absence of any prothrombotic risk factors other than malignancy, suggest that thalidomide was the agent for the arterial thrombosis in our patient. It is not known why this complication occurs despite the administration of anticoagulant prophylaxis. The role of factor VIII, von Willebrand factor antigen levels and fibrinogen in MM patients should be studied so these factors can be targeted in future prophylactic treatment.

\section{REFERENCES}

1. National Comprehensive Cancer Network. Clinical practice guidelines in oncology. Multiple myeloma. 2014. Available at: http://www.nccn.org/professionals/physician_gls/f guidelines.asp\#myeloma (accessed 5 Feb 2016).

2. Thompson M. Direct Healthcare Professional Communication on the association between Thalidomide CelgeneTM (thalidomide) and thromboembolism. 6 May 2011.

3. Schütt P, Ebeling P, Buttkereit U, Brandhorst D, Opalka B, Poser M, et al. Thalidomide in combination with dexamethasone for pretreated patients with multiple myeloma: serum level of soluble interleukin-2 receptor as a predictive factor for response rate and for survival. Ann Haematol 2005;84:594-600.

4. Haemato-oncology Task Force of the British Committee for Standards in Haematology (BCSH), UK Myeloma Forum. Guidelines for the diagnosis and management of multiple myeloma. 2014. Available at: http://www.bcshguidelines.com/documents/MYELOMA_GUIDELINE_Feb_2014_for_BCSH.pdf (accessed 1 September 2015).

5. Zonder J, Schiffer C A. Thrombotic complications following treatment of multiple myeloma with thalidomide and its analogues. 2014. Available at: http://www.uptodate.com/ contents/thrombotic-complications-following-treatment-of-multiple-myeloma-with-thalidomide-and-its-analogues?source=see_link (accessed 1 March 2016).

6. Bowcock SJ, Rassam SM, Ward SM, et al. Thromboembolism in patients on thalidomide for myeloma. Hematology 2002;7:51-53.

7. Ortin X, Rodriguez-Luaces M, Calabuig M, et al. Stroke in a multiple myeloma patient treated with thalidomide. J Stroke Cerebrovasc Dis 2006;15:283-285.

8. Altintas A, Ayyildiz O, Atay AE, et al. Thalidomide-associated arterial thrombosis: two case reports. Ann Acad Med 2007;36:304-306.

9. Goz M, Eren MN, Cakir O. Arterial thrombosis and thalidomide. J Thromb Thrombolysis 2008;25:224-226.

10. Jeng WJ, Kuo MC, Shih LY, et al. Pulmonary embolism in a patient with multiple myeloma receiving thalidomide-dexamethasone therapy. Int J Hematol 2008;87:542-544.

11. Ferri M, Faggioli G, Fratesi F, Stella A. Multifocal arterial thrombosis during thalidomide therapy: case report and review of the literature. Case Rep Med 2009;2009:372073.

12. Alkindi S, Dennison D, Pathare A. Arterial and venous thrombotic complications with thalidomide in multiple myeloma. Arch Med Res 2008;39:257-258.

13. Scarpace SL, Hahn T, Roy H, et al. Arterial thrombosis in four patients treated with thalidomide. Leuk Lymphoma 2005;46:239-242.

14. Libourel EJ, Sonneveld P, van der Holt B, et al. High incidence of arterial thrombosis in young patients treated for multiple myeloma: results of a prospective cohort study. Blood 2010;116:22-26.

15. Palumbo A, Palladino C. Venous and arterial thrombotic risks with thalidomide: evidence and practical guidance. Ther Adv Drug Saf 2012;3:255-266. 